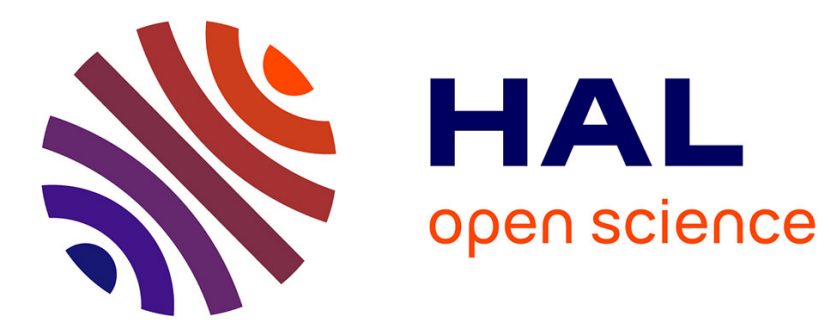

\title{
Fluid-structure interactions of a circular cylinder in a stratified fluid
}

\author{
Sarah Christin, Patrice Meunier, Stéphane Le Dizès
}

\section{To cite this version:}

Sarah Christin, Patrice Meunier, Stéphane Le Dizès. Fluid-structure interactions of a circular cylinder in a stratified fluid. Journal of Fluid Mechanics, 2021, 10.1017/jfm.2021.155 . hal-03372907

\section{HAL Id: hal-03372907 \\ https://hal.science/hal-03372907}

Submitted on 11 Oct 2021

HAL is a multi-disciplinary open access archive for the deposit and dissemination of scientific research documents, whether they are published or not. The documents may come from teaching and research institutions in France or abroad, or from public or private research centers.
L'archive ouverte pluridisciplinaire HAL, est destinée au dépôt et à la diffusion de documents scientifiques de niveau recherche, publiés ou non, émanant des établissements d'enseignement et de recherche français ou étrangers, des laboratoires publics ou privés. 


\title{
Fluid-structure interactions of a circular cylinder in a stratified fluid
}

\author{
Sarah Christin $^{1} \dagger$, Patrice Meunier ${ }^{1}$ and Stéphane Le Dizès ${ }^{1}$ \\ ${ }^{1}$ Aix-Marseille Université, CNRS, Centrale Marseille, IRPHE, Marseille, France
}

(Received xx; revised xx; accepted xx)

In this article, the objective is to characterize the influence of a continuous stratification on wake induced vibrations of a circular cylinder. Experimental results are obtained by towing cylinders, fixed to free-to-rotate arms, at a constant speed in a linearly stratified fluid made of salty water. The diameter of the cylinder, the length of the arms and the translating speed are varied. Two flow induced vibrations modes are observed. The first one is interpreted as a Vortex Induced Vibration (VIV) mode: it is associated with a resonance between the vortex shedding frequency and the natural frequency of the mechanical system. The cylinder oscillation and vortex shedding frequencies are also found to be locked in a large range of velocities in agreement with the low-mass-ratio VIV theory. The second mode is shown to be a galloping mode. It has a low frequency and a large amplitude, and occurs for low Froude numbers and long arms. It corresponds to the regimes when the buoyancy forces are larger than the inertial and vertical drag forces. By computing the forces on the cylinder, it is shown that stratification is the source of a destabilizing lift when the cylinder departs from its horizontal motion. Only a weak effect of the Reynolds number on the stability characteristics has been observed in the considered range $(500<R e<15000)$.

\section{Introduction}

Despite the very large number of studies on Flow Induced Vibrations (FIV), none of them considered the case of horizontal wakes in a stratified fluid. This work aims to characterize the influence of a continuous stratification on wake induced vibrations of a circular cylinder.

Many works have concerned simple geometries such as circular cylinder that exhibit a large variety of regimes and wake structures. They were initially motivated by applications such as marine cables (Griffin 1985), or taut wires in air (Strouhal 1878). In this civil engineering context, it is important to minimize or control any undesired vibrations that could damage the structure. For other applications, one could instead be interested in increasing the oscillations as they could be a source of energy when coupled to piezoelectric devices (Bernitsas et al. 2008; Grouthier et al. 2014; Song et al. 2015).

As now documented in text books (Tritton 2012) and review articles (Williamson 1996b), the wake of a cylinder is known to exhibit different regimes as the Reynolds number is varied. As the Reynolds number increases, one observes successively the breaking of the upstream/downstream symmetry, the appearance of a separation bubble (Coutanceau \& Bouard 1977), vortex shedding (Von Karman 1911), and a series of 3D transitions (Williamson 1996a). One of the most remarkable features is the persistence of the von Karman vortex street for Reynolds numbers as large as $10^{7}$ (Roshko 1961). These coherent structures induce coherent pressure fluctuations on the cylinder that are

$\dagger$ Email address for correspondence: sarah.christin@univ-amu.fr 
responsible for lift and drag fluctuations. When the cylinder is free to move, this induces transverse oscillations (Feng 1968): this is the so-called Vortex Induced Vibrations (VIV). Reviews can be found in Bearman (1984); Williamson \& Govardhan (2004); Sarpkaya (2004).

VIV has generally been studied experimentally by mounting a circular cylinder on springs in a water channel or a wind tunnel. The system then possesses two natural frequencies:

- The frequency associated with the transverse displacement of the structure given by the natural frequency of the springs :

$$
f_{n}=\sqrt{\frac{k}{\left(m+m_{A}\right)}}
$$

where $k$ is the spring constant, $m$ the cylinder mass and $m_{A}$ the potential added mass. - The frequency associated with vortex shedding given for a fixed cylinder by an empirical formula:

$$
f_{V}=S t \frac{U}{D}
$$

where $S t$ is the Strouhal number (Strouhal 1878), $U$ the fluid velocity and $D$ the cylinder diameter. $S t$ is found experimentally to be close to 0.2 in a large range of Reynolds numbers $200<R e<200000$ (Lienhard 1966).

In the classical VIV theory (reviewed by Williamson \& Govardhan 2004, for instance), the vortex shedding frequency is found to synchronize with $f_{n}$ when $f_{V}$ reaches $f_{n}$ (Den Hartog 1954). Both the structure oscillations and vortex shedding then remain locked in at almost a constant frequency in a large interval of velocities. For large mass ratio $m^{*}$ (equal to the cylinder mass $m$ divided by the mass of an equivalent volume of fluid), this frequency is close to $f_{n}$, as observed in the experiments in air performed by Feng (1968). For smaller $m^{*}$ (Khalak \& Williamson 1999), this mode of constant lock-in frequency is again observed, called the "lower branch mode", but with higher amplitudes and a larger frequency compared to high-mass-ratio experiments. This lockin frequency has been determined empirically by Govardhan \& Williamson (2000) for a circular cylinder as:

$$
f_{\text {lower }}^{*}=\sqrt{\frac{m^{*}+C_{A}}{m^{*}-0.54}},
$$

where the added mass coefficient $C_{A}$ is set to 1 . Furthermore, in this low $m^{*}$ case, a second "upper branch mode" is observed. It results in oscillations of even larger amplitude than the first mode. The oscillations start when $f_{V}$ reaches $f_{n}$, and have a frequency which grows with the velocity up to $f_{\text {lower }}^{*}$, where the system switch to the lower branch mode.

Another type of flow induced vibration can also come from the breaking of the axisymmetry of the object, it is called galloping. When the object is not circular, a small angle of incidence may induce a destabilizing lift force. This leads to high amplitude low frequency oscillations that have been studied for rectangular cylinders (Mannini et al. 2014), and modified circular cylinder geometries (Chang et al. 2011; Nakamura et al. 1994). Some studies have also considered the interaction of galloping and vortex shedding (Parkinson \& Wawzonek 1981; Mannini et al. 2016). Note finally that there exist other FIV phenomena such as buffeting (Blevins 1977), or resulting from the interaction of several objects (Assi et al. 2010). 
Studies of transverse FIV have considered very complex systems but surprisingly have never tried to include stratification, while most fluids in the environment are stratified. A stably stratified fluid has a density that increases with depth. It possesses an intrinsic natural frequency, the Brünt-Väisälä frequency $N$ defined by

$$
N^{2}=-\frac{g}{\rho_{0}} \frac{\partial \rho}{\partial z}
$$

where $\rho$ is the fluid density, $\rho_{0}$ is a reference density, $g$ the gravity along the vertical direction $z$. This frequency corresponds to the natural oscillation frequency of a fluid parcel when vertically displaced from its equilibrium position. This new additional frequency in the system is expected to modify the VIV phenomena described above.

Stratification is already known to modify the wake of a fixed cylinder. As it inhibits vertical motion, it delays the appearance of vortex shedding and narrows the vertical extent of the wake (e.g. Lin \& Pao 1979). It can also be responsible for the appearance of a second vortex shedding mode when the cylinder is tilted with respect to the horizontal plane (Meunier 2012). The reference experimental study for an horizontal circular cylinder has been carried out by Boyer et al. (1989). They have been able to observe 10 different types of regimes as the Reynolds number and the stratification strength are varied. Even more regimes have been found in Chashechkin \& Voyekov (1993).

In the present work, we start exploring experimentally the new field of "stratified wake induced vibrations" for the simple and most documented geometry of a circular cylinder in a large Reynolds number regime. The objective is to evidence some new phenomena, describe their characteristics and tentatively provide some physical explanations.

The paper is organized as follows. In section $\S 2$, the experimental set-up is first described. The main control parameters of the problem are identified. Reference data with a fixed cylinder are also obtained. In section $\S 3$, the FIV results are presented. Two oscillation modes are obtained which are successively discussed in sections $\S 4$ and $\S 5$. In section $\$ 5$, some numerical computations are also performed to identify the mechanism of instability. In section $\S 6$, the competition between the two modes is further studied, to propose a stability diagram for the system. A brief conclusion is made in section $\S 7$.

\section{A stratified VIV experiment}

\subsection{Facility}

The experimental setup, sketched in figure 1, consists of a PMMA tank $4 \mathrm{~m}$ long, 1 $\mathrm{m}$ wide and $1 \mathrm{~m}$ deep, which is filled with salty water up to a height $H$ between 70 and $85 \mathrm{~cm}$. A trolley is mounted on rails and moved with a ball screw connected to a motor MAC800 D2 of JVL, allowing a horizontal translation of the system along the tank. The velocity of the trolley is kept constant during each run and vary in the range $0.01 \leqslant U \leqslant 23 \mathrm{~cm} \cdot \mathrm{s}^{-1}$.

Three different cylinders of diameter $D$ (equal to $3.2,4$ and $6.2 \mathrm{~cm}$ ) and length $L_{y}$ of $63.9 \mathrm{~cm}$ are considered. This leads to large ratios $L_{y} / D>10$ and $H / D>12$ which minimizes end effects in the transverse direction $y$ and at the top and bottom of the tank. As illustrated in figure 1, the cylinder is mounted on flat blade-shaped arms, which are free to rotate around their junction point with the supports thanks to ball bearings. The length $L$ of these arms is an important parameter chosen between 15 and $50 \mathrm{~cm}$. Their flat shape and junction with the cylinder using two screws limit tilting and minimize the arm-induced drag. All the parameters of the experiments are summarized in table 1 . 


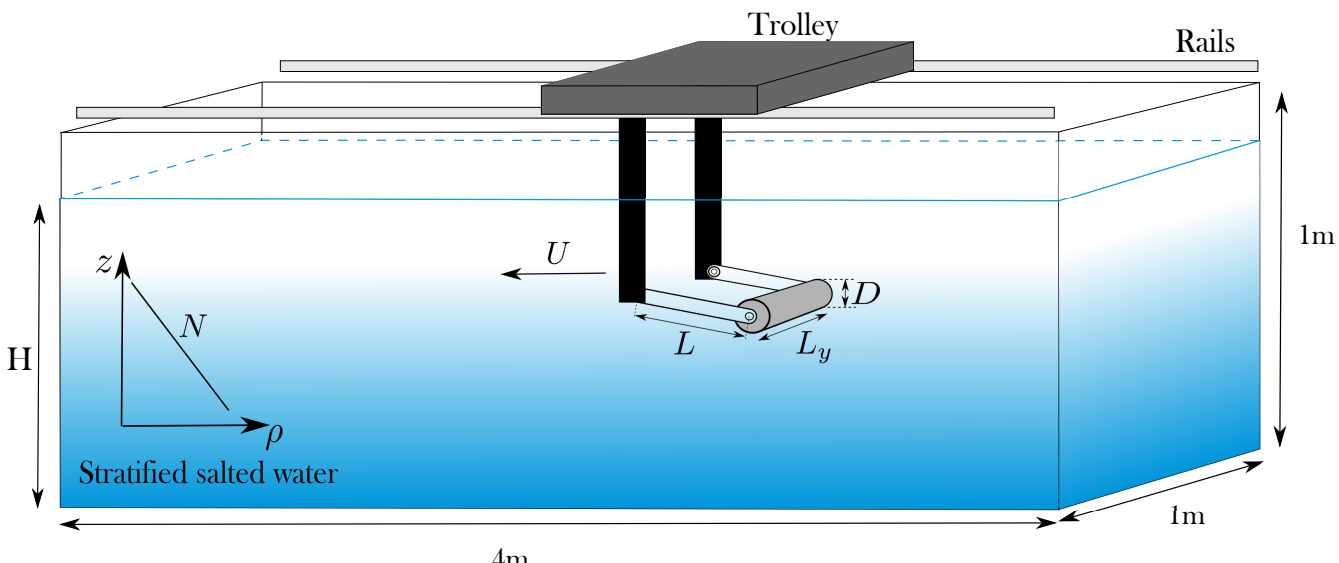

FIGURE 1. Sketch of the experimental setup with the main parameters: velocity $U$, length of arms $L$, cylinder diameter $D$ and the Brünt Väisälä frequency $N$ defined by equation (1.4)

\begin{tabular}{|c|c|c|c|c|c|c|c|}
\hline Exp. $n^{\circ}$ & $D[\mathrm{~cm}]$ & $L[\mathrm{~cm}]$ & $N\left[\operatorname{rad} . \mathrm{s}^{-1}\right]$ & $l$ & $R e / F r$ & $U_{0}^{*}$ & Symbol \\
\hline 1 & & 15 & $0.4 \pm 0.1$ & 2.42 & $1.5410^{3}$ & 17.32 & $\star$ \\
\hline 2 & 6.2 & 30 & $0.52 \pm 0.05$ & 4.84 & $2.0010^{3}$ & 24.50 & 0 \\
\hline 3 & & 50 & $0.72 \pm 0.02$ & 8.06 & $2.7710^{3}$ & 31.62 & $\square$ \\
\hline 4 & & 15 & $0.37 \pm 0.02$ & 3.75 & $5.9210^{2}$ & 21.57 & $\star$ \\
\hline 5 & 4 & 30 & $0.62 \pm 0.03$ & 7.5 & $9.9210^{2}$ & 30.50 & 0 \\
\hline 6 & & 50 & $0.65 \pm 0.05$ & 12.5 & $1.0410^{3}$ & 39.37 & $\square$ \\
\hline 7 & 4 & 15 & 0 & 3.75 & 0 & 21.57 & $\star$ \\
\hline 8 & & 15 & $0.45 \pm 0.1$ & 4.67 & $4.6110^{2}$ & 24.07 & $\star$ \\
\hline 9 & & 19 & $0.95 \pm 0.01$ & 6 & $9.6910^{2}$ & 27.28 & $\diamond$ \\
\hline 10 & & 30 & $0.83 \pm 0.06$ & 9.38 & $8.5010^{2}$ & 34.12 & 0 \\
\hline 11 & 3.2 & 30 & $0.38 \pm 0.05$ & 9.38 & $3.8910^{2}$ & 34.12 & 0 \\
\hline 12 & & 34 & $1.07 \pm 0.15$ & 11 & $1.0910^{2}$ & 36.94 & * \\
\hline 13 & & 45 & $1.22 \pm 0.07$ & 14 & $1.2310^{2}$ & 41.67 & + \\
\hline 14 & & 50 & $0.83 \pm 0.06$ & 15.63 & $8.5010^{2}$ & 44.03 & $\square$ \\
\hline
\end{tabular}

TABle 1. Parameters of the different experimental configurations. For each configuration, about 20 different values of the translation velocity are considered between $0.01 \mathrm{~cm} / \mathrm{s}$ and $23 \mathrm{~cm} / \mathrm{s}$. The last column indicates the symbol used for each experiment in the figures.

The damping induced by ball bearings is measured in air using the pendulum model:

$$
\ddot{\theta}+\frac{c}{m} \dot{\theta}+\omega_{0}^{2} \theta=0
$$

with $\omega_{0}=\sqrt{g / L} \approx 4.43 \mathrm{rad} . \mathrm{s}^{-1}$ for the arms of length $L=50 \mathrm{~cm}$. The damping coefficient $c$ is found by fitting the exponential decay of the cylinder position over 96 periods, and found to be $c=5.6 \pm 0.210^{-3} \mathrm{~kg} \mathrm{~s}^{-1}$.

The mass $m$ of the cylinder is finely adjusted so that it is at equilibrium when the 


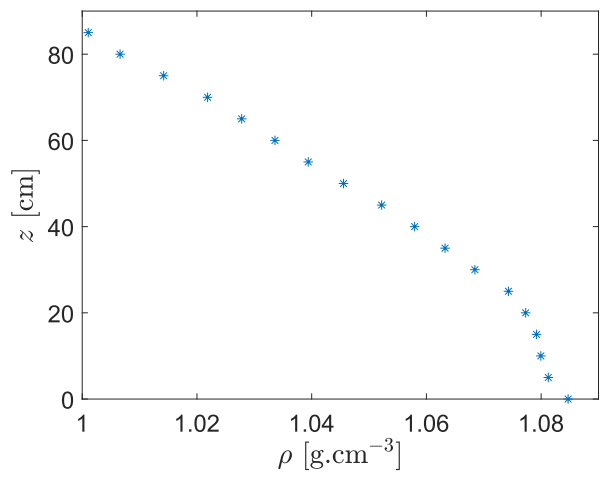

Figure 2. Plot of the density profile two days after the creation of the stratification. By taking $\rho_{0}=\rho(z=50 \mathrm{~cm})=1.046 \mathrm{~g} . \mathrm{cm}^{-3}$ as density reference, one can find $N=1.06 \mathrm{rad} . \mathrm{s}^{-1}$.

arms are horizontal. The uncertainties are such that, at rest, the cylinder is at $50 \pm 4 \mathrm{~cm}$ from the bottom of the tank.

\subsection{Stratification}

The tank is filled with salty water with a salt concentration increasing linearly with depth. The fluid density can be written as $\rho=\rho_{0}\left(1-z N^{2} / g\right)$ where $\rho_{0}$ is the density at the cylinder equilibrium position.

This stratification is obtained using a technique described in the $\mathrm{PhD}$ thesis of Bosco (2015). It consists in dividing the tank into two parts, one with pure water and the other one with uniformly mixed strongly salty water, and letting the two fluids mix through holes of diameter $0.5 \mathrm{~cm}$ drilled in the separation slab. This technique is preferred to the usual "two tanks method" because it requires only one tank, which is far more convenient considering the huge volume used here.

A plot of the density profile just after the stratification creation is shown in figure 2 . One can notice that the profile is linear from $z=20 \mathrm{~cm}$ to the free surface at $z=85 \mathrm{~cm}$, but a mixed layer is observed for $z<20 \mathrm{~cm}$. This is due to a stronger mixing occurring at the bottom when the salty and pure water blend through the slab holes. We have been able to limit this effect that could also happen at the free surface by closing some of the holes.

Experiments are typically performed during a few months with the same water. During this period, the value of $N$ decreases progressively owing to leaks and the mixing generated by the displacements of the cylinder. In order to minimize these effects, the tank is regularly filled with fresh water at the top and/or salty water at the bottom. Every experimental day, a density profile is measured to recompute $N$, but mixing still induces uncertainties on $N$ from $2 \%$ up to $25 \%$ for the runs with the largest cylinder diameter and the strongest oscillations.

\subsection{Important non dimensional parameters}

From the geometrical parameters $\left(D, L, H, L_{y}\right)$, as defined in figure 1 , the translation speed $U$ and the fluid properties (kinetic viscosity $\nu$, diffusion coefficient $\mathcal{D}$, and $N$ ), an important number of non-dimensional parameters can be defined:

- the Reynolds number $R e=U D / \nu$,

- the Froude number $F r=U / N D$,

- the length ratio $l=L / D$, 


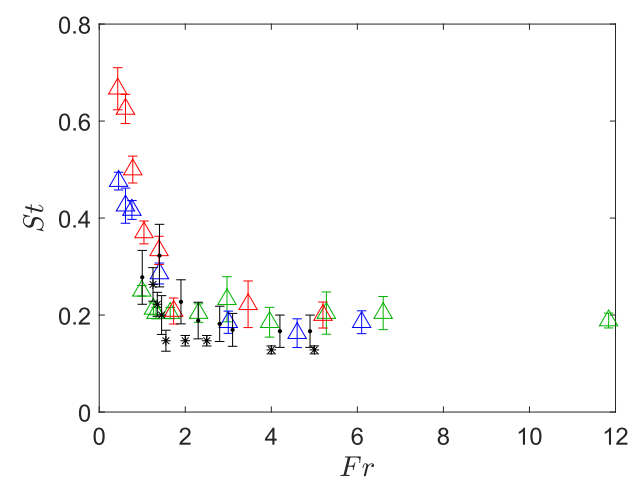

Figure 3. Strouhal number as a function of the Froude number. $D=2.1 \mathrm{~cm}$ (green), $D=3.1 \mathrm{~cm}$ (blue) and $D=4.1 \mathrm{~cm}$ (red). Black symbols stand for experimental (stars) and numerical (dots) results of Meunier (2012) $(R e \in[40,200])$.

- the Schmidt number $S c=\nu / \mathcal{D}$,

- the mass ratio $m^{*}=m /\left(\rho_{0} L_{y} \pi D^{2} / 4\right)$,

- the geometric ratios $L_{y} / D$ and $H / D$

These last two ratios are large. For this reason, end effects as well as boundary effects associated with the free surface, side and bottom walls are assumed to be negligible. They are not considered in the present study. The Schmidt number is close to $S c \approx 625$ obtained for $\nu=10^{-6} \mathrm{~m}^{2} \mathrm{~s}^{-1}$ and $\mathcal{D}=1.6410^{-9} \mathrm{~m}^{2} \mathrm{~s}^{-1}$. The mass ratio $\mathrm{m}^{*}$, is here always equal to 1 , which is much smaller than in classical VIV experiments where $m^{*}$ is larger than 20 most of the time.

In the present study, the three important parameters are then $R e, F r$ and $l$. They will be varied in the following ranges: $500<R e<15000 ; 0.5<F r<19 ; 2<l<16$. Of interest is also the ratio $R e / F r$ that remains constant for each experiment number indicated in table 1. Except for the non-stratified case (experiment number 7 ), this ratio is large, indicating the dominance of buoyancy over viscous effects.

\subsection{Measurements with fixed cylinder: the Strouhal number}

The Strouhal number, defined in equation (1.2), corresponds to the non dimensional frequency associated with vortex shedding for a fixed cylinder. There are only a few data for the Strouhal number law in a stratified fluid. For this reason, experiments with a fixed cylinder are first performed in order to find the Strouhal number dependence in the setup.

Three different cylinders of diameter $D=2.1,3.1$ and $4.1 \mathrm{~cm}$ are considered. The measurements were made by analysing the wake patterns in the last two meters of the cylinder course with a shadowgraph method using a camera Sony $\alpha 7 \mathrm{~s}$ taking 25 frames per second, and lens FE 2.8/50 MACRO. Most patterns were already qualitatively described in Boyer et al. (1989).

The frequency was measured and dimensionalized to obtain the Strouhal number. Results are plotted in figure 3 as a function of the Froude number, which offers the best collapse.

The Reynolds number is equal to $R e=410 \mathrm{Fr}$ for $D=2.1 \mathrm{~cm}$, to $R e=894 \mathrm{Fr}$ for $D=3.1 \mathrm{~cm}$, to $R e=1563 \mathrm{Fr}$ for $D=4.1 \mathrm{~cm}$. The Reynolds number ranges from 400 to 8000 in these experiments. On this plot is also displayed the experimental and numerical results obtained by Meunier (2012) for lower Reynolds numbers $(40<R e<200)$. This 
figure clearly shows that the usual value $S t \approx 0.2$ of the homogeneous case remains valid here as soon as $\mathrm{Fr}>1.5$.

For lower $F r$, a sudden increase of the vortex shedding frequency is observed, in agreement with the numerical and experimental results of Meunier (2012). We can also see that this increase becomes larger when the diameter of the cylinder is increased for a fixed Fr. This means a growth of the Strouhal number with the Reynolds number for small fixed $\mathrm{Fr}$. This tendency is in agreement with the results obtained by Chashechkin \& Voyekov (1993) who observe a linear increase of $S t$ with $R e$ for small Froude numbers.

\subsection{Measurements with oscillating cylinder and sources of uncertainty}

As for the fixed cylinder, FIV measurements were performed in the last two meters of the cylinder course with the same apparatus. The oscillations of the cylinder are characterized by monitoring with the software Tracker the angle $\theta$ made by the arms with respect to the horizontal plane. From a least squares fit of the time variation of the angle, an amplitude $A$ and a frequency $f$ are obtained.

Sources of uncertainties coming from the visualisation method or the stratification measurement for instance, are numerous. A few of them can be quantified.

Uncertainties on the Froude number $\mathrm{Fr}$ come from the variation of $N$ due to mixing. As already mentioned, they can reach $20 \%$. Uncertainties on the Reynolds number Re of about $10 \%$ are mainly due to temperature variation, which modifies the value of the kinematic viscosity. Uncertainties on the oscillation frequency of the higher-frequency mode (see section 4) are of order $5 \%$, and those on the amplitudes of order $20 \%$, mainly due to the irregularities of the observed oscillations. For the low-frequency modes, the uncertainties are of order $25 \%$ for both amplitude and frequency measurements because of numerous bias. The first one is due the limited length of the tank: only part of the very long wavelength oscillations can be observed and it is difficult to guarantee that a steady regime has been reached. The second one is associated with the stratification. Although a particular attention has been brought by waiting for at least 15 minutes between experimental runs, stratification remains slightly perturbed by the cylinder displacements. This probably explains the dispersion of the amplitude measurements that have been observed between the first and last run of an experimental day.

\section{The existence of two vibration modes}

Videos of the cylinder moving through the tank were analyzed to deduce the evolution of vertical position $z$ of the cylinder as a function of time. A plot of these raw data, in a homogeneous and a stratified fluid, for fixed $l$ and $R e$, is shown in figure 4 . It is clear that the cylinder exhibits strong oscillations in a stratified fluid whereas oscillations are almost undetectable in a homogeneous fluid. This is a first indication that stratification strongly affects the flow induced vibrations of a cylinder.

Measurements of the amplitude and frequency of the cylinder oscillations are reported in figure 5 as a function of the Reynolds number for the homogeneous and stratified cases. It confirms that although oscillations are observed in both cases, their amplitude in stratified water are more than one decade higher than in a homogeneous fluid. Their frequencies seem to follow the same slope, but they are higher in the homogeneous case.

The stratified experiments were repeated with different speeds $U$, cylinder diameters $D$ and arm's lengths $L$ to explore the space of the parameters $F r, R e$ and $l$.

Data of measured amplitudes $A$ and frequencies $f$ obtained for a fixed value of $l$ are shown in figure 6 . One can see that, as $F r$ increases, the cylinder firstly exhibits large amplitude vibrations at low frequency (with an amplitude peak around $F r=1.5$ ), 


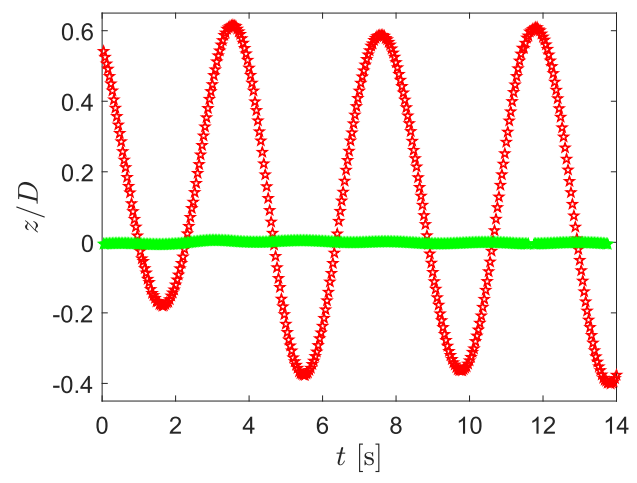

FiguRE 4. Vertical position of the cylinder in a homogeneous flow (green) and a weakly stratified flow with $F r=6.16$ (red). $R e=4158, l=3.75$.

(a)
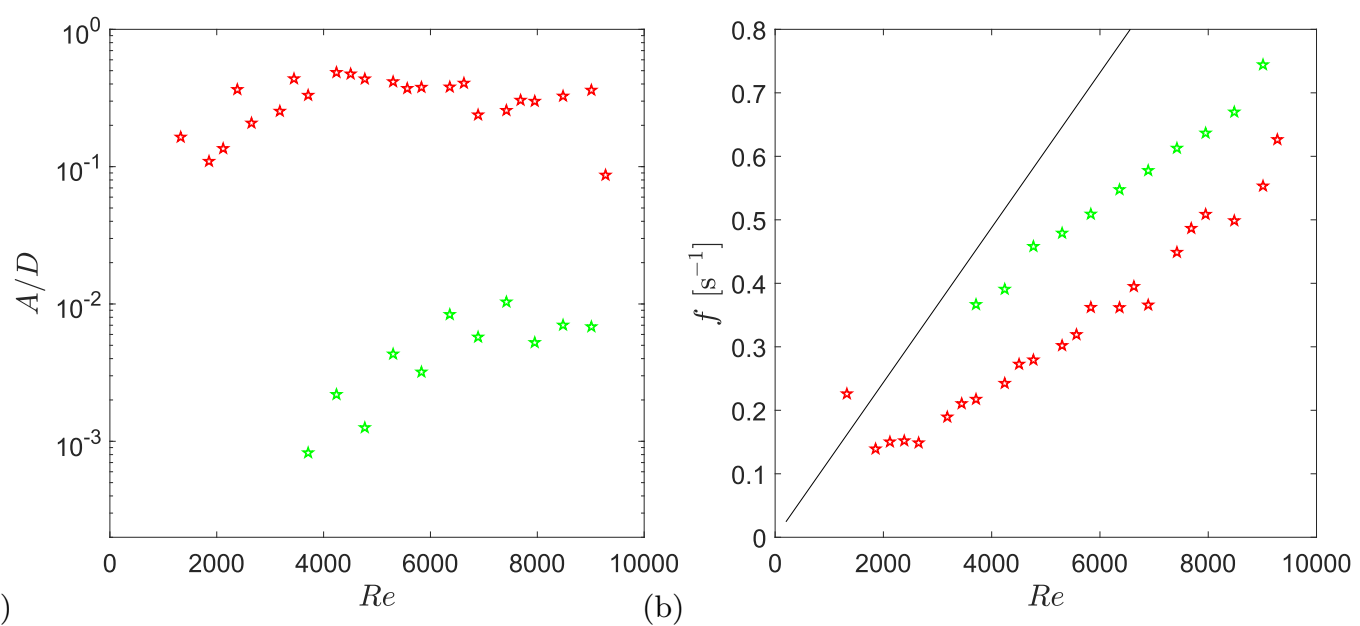

FiguRE 5. Amplitude (a) and frequency (b) of the cylinder oscillations as a function of the Reynolds number in a stratified (red) and homogeneous (green) fluid for $l=3.75$. Black line represents the Strouhal frequency $(S t=0.2)$. In the stratified case, $F r$ varies from 1.8 for the smaller Reynolds number to 12.6 for the larger Reynolds number.

then oscillations almost disappear before increasing again, to a lesser extent, while their frequency jumps to a value about six times their initial value. This indicates the existence of two distinct vibration modes.

These two modes are also clearly visible in figure 7 where is plotted as a function of $\mathrm{Fr}$ the measured frequency $f$ for all studied $l$. For visibility reasons, only frequency measurements for which the oscillation amplitude was larger than $0.03 D$ are kept. This amplitude threshold has also been applied to all the following frequency plots, except in the final plots of section $\S 6$. The large amount of data extending from low $F$ less than one up to $F r=20$ enables to better identify the two frequency modes already spotted on figure 6 . The first mode has low frequency of order $0.25 \mathrm{~N} / 2 \pi$; it is obtained for small Froude numbers between 1 and 3 and is favored by large $l$. The second mode has a higher frequency that increases with $U$.

In the next two sections, a focus is made on each frequency branch to detail the observations and provide some physical interpretations. 

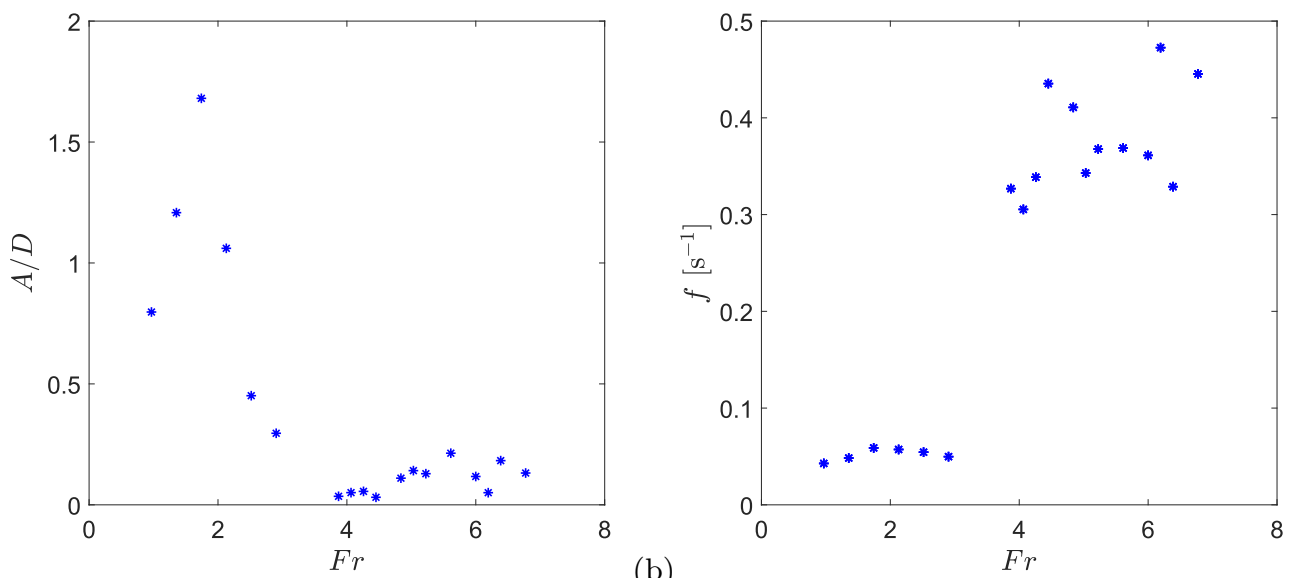

(a)

(b)

Figure 6. Amplitude (a) and frequency (b) of the cylinder oscillations as a function of the Froude number for one set of cylinder diameter and length of arms $(l=11)$.

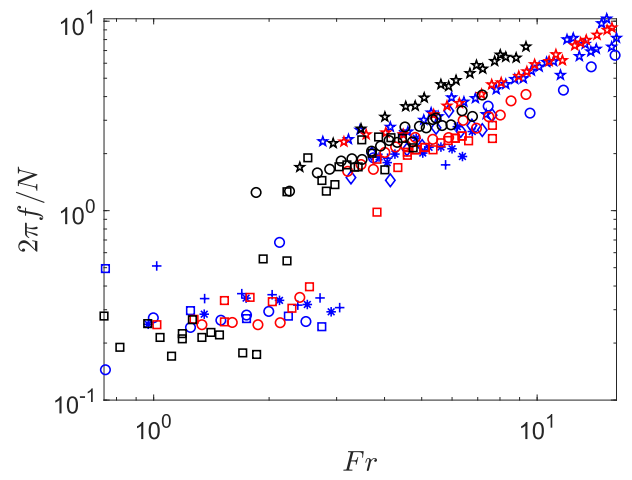

Figure 7. Frequency of the oscillation as a function of the Froude number for all $l$ considered. Black, red and blue symbols stand for the larger, intermediate and smallest cylinder diameters, respectively (see table 1). Only experiments with amplitude oscillations larger than $0.03 D$ are plotted.

\section{The VIV mode}

The high-frequency mode is firstly considered. The objective is to show that this mode corresponds to a classical VIV mode. For this purpose, a simple mathematical model is derived to estimate the natural frequency $f_{n}$ of the mechanical system.

The model is based on the application of the momentum equation to the cylinder assuming that the forces acting on the rod can be neglected (small mass, negligible drag). For a cylinder of density $\rho_{0}$, volume $V_{s}$, diameter $D$ and length $L_{y}$, supported by a rigid rod of length $L$ which makes an angle $\theta$ with respect to the horizontal plane (see figure 8), the following forces acting on the cylinder can be listed:

- Rod's tightness $\boldsymbol{F}_{\boldsymbol{T}}$

- Weight $\boldsymbol{F}_{\boldsymbol{w}}=\rho_{0} V_{s} \boldsymbol{g}$

- Buoyancy force $\boldsymbol{F}_{\boldsymbol{b}}=-\rho(\theta) V_{s} \boldsymbol{g}$

- Drag force $\boldsymbol{F}_{\boldsymbol{D}}=-\frac{1}{2} \rho(\theta) D L_{y} C_{D} U_{\mathrm{abs}} \boldsymbol{U}_{\mathbf{a b s}}$

- Lift force $\boldsymbol{F}_{\boldsymbol{L}}=-\frac{1}{2} \rho(\theta) D L_{y} C_{L} U_{\text {abs }}\left(\boldsymbol{e}_{\boldsymbol{y}} \wedge \boldsymbol{U}_{\text {abs }}\right)$ 


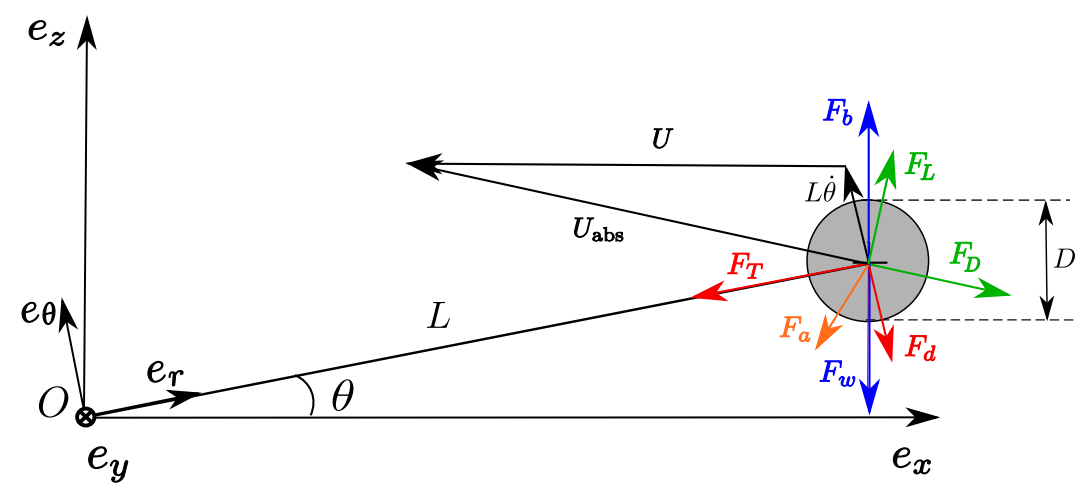

FIgURE 8. Schematic of the problem. Opposite or perpendicular forces are colored by pair to ease comprehension.

- Added mass force $\boldsymbol{F}_{\boldsymbol{a}}=-C_{A} \rho(\theta) V_{s} L\left(\ddot{\theta} \boldsymbol{e}_{\boldsymbol{\theta}}-\dot{\theta}^{2} \boldsymbol{e}_{\boldsymbol{r}}\right)$

- Ball bearing damping $\boldsymbol{F}_{\boldsymbol{d}}=-c L \dot{\theta} \boldsymbol{e}_{\boldsymbol{\theta}}$

where $C_{D}$ and $C_{L}$ denote the drag and lift coefficients, respectively, and $C_{A}$ the added mass coefficient for a circular cylinder and $\rho(\theta)=\rho_{0}\left(1-N^{2} L \sin (\theta) / g\right)$ the density of the ambient fluid at the cylinder position. Figure 8 shows these forces and their orientation. The absolute velocity $\boldsymbol{U}_{\text {abs }}$ is the effective velocity of the cylinder due to the combined action of its translation by the trolley and its rotation around the ball bearing caused by oscillations, so that $\boldsymbol{U}_{\text {abs }}=-U \boldsymbol{e}_{\boldsymbol{x}}+L \dot{\theta} \boldsymbol{e}_{\boldsymbol{\theta}}$. By definition, the drag force is oriented in the direction of $-\boldsymbol{U}_{\text {abs }}$ while the lift force is perpendicular to it.

Applying the operation $\boldsymbol{e}_{\boldsymbol{r}} \times$ to the momentum equation leads to the angular momentum equation:

$V_{s}\left(\rho_{0}+\rho C_{A}\right) L \ddot{\theta}+c L \dot{\theta}=\frac{F_{D}}{U_{\mathrm{abs}}}[U \sin (\theta)+L \dot{\theta}]-\frac{F_{L}}{U_{\mathrm{abs}}} U \cos (\theta)+\left(\rho_{0}-\rho\right) V_{s} g \cos (\theta)$.

By assuming $\theta \ll 1, L \dot{\theta} \ll U, C_{L} \ll 1, C_{A}=1$, keeping only the first order terms in equation (4.1), and switching from $\theta$ to $z=L \theta$, the following equation is obtained:

$$
\ddot{z}+\left(\frac{c}{2 \rho_{0} V_{s}}+\frac{C_{D} U}{\pi D}\right) \dot{z}+\left(\frac{N^{2}}{2}+\frac{C_{D} U^{2}}{\pi L D}\right) z=\frac{C_{L} U^{2}}{\pi D} .
$$

This is the classical equation of a forced damped oscillator, that is forced by the lift and damped by ball bearings and drag force.

Equation (4.2) shows that the restoring forces due to drag and stratification combine to create an oscillator of natural frequency:

$$
f_{n}=\frac{1}{2 \pi} \sqrt{\frac{N^{2}}{2}+\frac{C_{D} U^{2}}{\pi L D}} .
$$

This formula applies to both homogeneous and stratified cases. The system is analogue to the usual VIV system where the cylinder is mounted on springs. In the absence of stratification, its natural frequency is $f_{n}=(U / 2 \pi D) \sqrt{C_{D} / \pi l}$ which has to be compared to the natural frequency $f_{n}=(1 / 2 \pi) \sqrt{k / m}$ associated with springs. The main difference is then the linear dependence of the natural frequency with respect to the velocity whereas it is constant for springs. The non dimensional velocity

$$
U^{*}=\frac{U}{f_{n} D}
$$


that is commonly used in VIV studies (Williamson \& Govardhan 2004), is thus constant and equal to

$$
U_{0}^{*}=2 \pi^{3 / 2} \sqrt{l / C_{D}}
$$

in the non-stratified case. For a cylinder $\left(C_{D}=1\right)$ and the parameters of the experiment (number 7 in table 1 ), $U_{0}^{*} \approx 21.5$. This value is very large compared to the value $1 / S t=5$ where VIV high amplitudes oscillations are expected in the classical theory (see section 1). In other words, the natural mechanical frequency and the vortex shedding frequency are both proportional to the velocity which implies that they never match for any velocity. It follows that no resonance is possible and no significant oscillations are thus expected in the homogeneous system, which is in agreement with what has been observed in figure 5 .

In the presence of stratification, the restoring force is due to the combined action of drag and stratification. The natural frequency of the system is thus no longer proportional to $U$ and for strong stratification $f_{n}$ becomes constant $f_{n} \sim N /(2 \sqrt{2} \pi)$. The expression of $U^{*}$ becomes for the general case

$$
U^{*}=\frac{2 \pi}{\sqrt{\frac{1}{2 F r^{2}}+\frac{C_{D}}{\pi l}}} .
$$

This value is bounded by $U_{0}^{*}$ that is reached for large Froude number. In table 1, the value of $U_{0}^{*}$ has been provided for each experimental configuration, using $C_{D}=1$.

Equation (4.2) provides the natural frequency $f_{n}$ but also the oscillator damping rate $\sigma_{D}$ as

$$
\sigma_{D}=\frac{1}{2}\left(\frac{c}{2 \rho_{0} V_{s}}+\frac{C_{D} U}{\pi D}\right) .
$$

Measures in still air demonstrate that the damping rate caused by the structure $\left(c /\left(2 \rho_{0} V_{s}\right) \approx 510^{-3} \mathrm{~s}^{-1}\right)$ is at least 20 times smaller than the drag damping term $\left(C_{D} U /(\pi D) \geqslant 0.1 \mathrm{~s}^{-1}\right) . \sigma_{D}$ thus reduces to the second term in (4.7). The damping ratio $\zeta=\sigma_{D} /\left(2 \pi f_{n}\right)$ is thus close to $U^{*} / 4 \pi^{2}$, and therefore always smaller than $U_{0}^{*} / 4 \pi^{2}$ which is at most equal to 1 .

In figure $9(\mathrm{a}), f / f_{n}$ is plotted versus $\mathrm{Fr} . C_{D}=1$ has been used, as always done in the following. One can see that the upper branch mode now exhibits a constant lock-in frequency around $2 f_{n}$ which confirms the link between the observed frequency and the natural frequency of the oscillator. This result is in excellent agreement with the formula (1.3) for the lock-in frequency of the lower branch VIV mode which predicts $f_{\text {lower }}^{*} \approx 2.1$ in the case of low mass ratio. This is clearly visible in figure 9(b) where this value of the frequency has been indicated in dashed line. This is a strong indication that the highfrequency mode does correspond to a VIV mode. In this figure where $f / f_{n}$ is plotted versus $U^{*}$ is also shown the vortex shedding frequency (in solid line). A synchronization on the right side of this line is observed as expected, but for larger values of $U^{*}$ than in classical VIV theory. However, the large extent of the synchronization interval is not far from the results obtained by Govardhan \& Williamson (2000) which predicts a lock-out at $U_{\text {end }}^{*} \approx 19.3$. It is also not in contradiction with the value of the damping ratio $\zeta$ which remains smaller than 1 for all the regimes where oscillations were observed.

In figure 10, the amplitude of the most unstable configurations is plotted as a function of $U^{*}$. These configurations correspond to the smallest values of $l$, for which the maximal value $U_{0}^{*}$ of $U^{*}$ is also the smallest. These limits are indicated as vertical dashed lines in the figure. The value of the peak amplitude decreases as $U^{*}$ increases as expected. But very low amplitudes are also observed for small $U^{*}$ that cannot be explained by the 
(a)

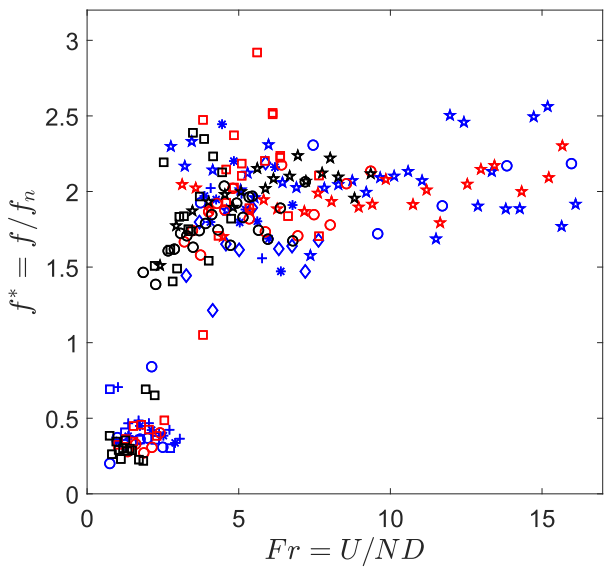

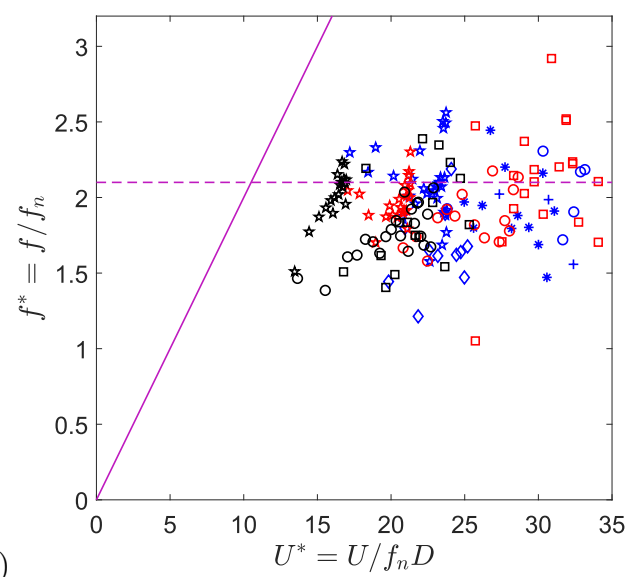

(b)

Figure 9. Frequency of VIV mode as a function of Froude number (a) and $U^{*}$ (b). Purple line stands for the Strouhal frequency $(S t=0.2)$. Purple dotted line stands for $f_{\text {lower }}^{*}$ as defined in equation (1.3). In (b) the lower frequency mode was removed for more clarity.

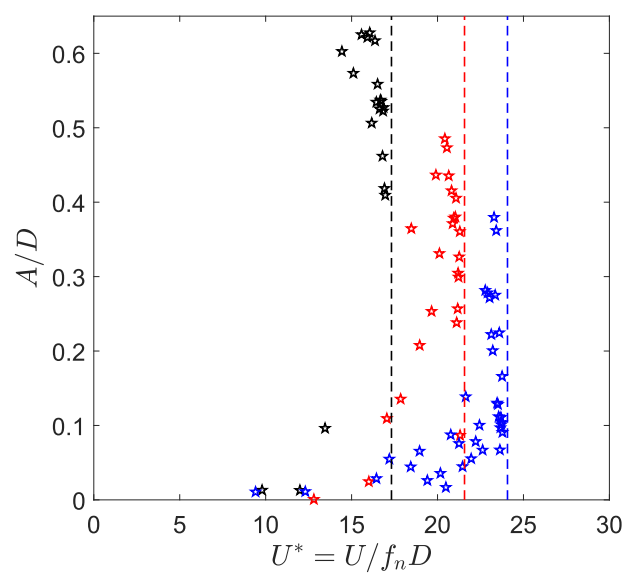

FIgURE 10. Amplitude of VIV mode as a function of $U^{*}$, for experiments with smaller $l$.

classical VIV theory. It is suspected that in this regime for which the Froude number is small, the stratification modifies the flow and the drag and lift forces it exerts on the cylinder.

\section{The galloping mode}

\subsection{Description and interpretation}

Below the VIV frequency branch, figure 7 shows the existence of a second vibration mode of the system with a constant frequency around $0.25 N / 2 \pi$. This mode occurs at rather low Froude numbers $(F r \in[1 ; 3])$, i.e. when the buoyancy forces are no longer negligible with respect to the inertial forces. Furthermore, it is not observed for small arms, but only when the ratio $l$ is larger than a critical value (around 7 ) as it will be further discussed in the next section. This indicates that the predominance of buoyancy forces over restoring drag forces is also probably important to observe this mode. 


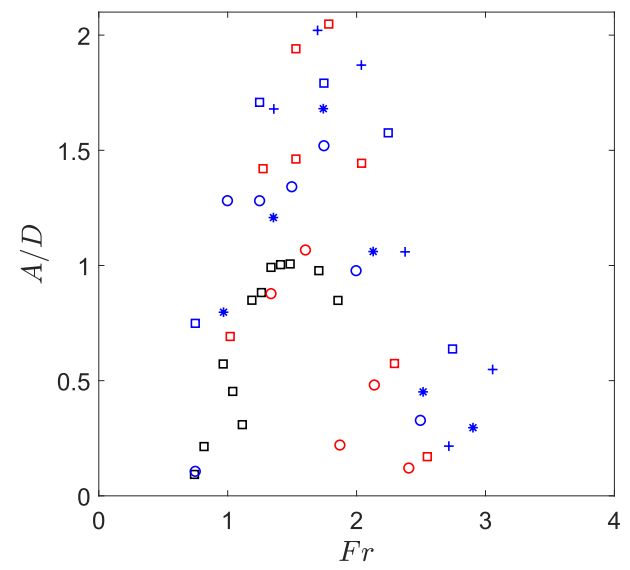

Figure 11. Amplitude of cylinder oscillation in galloping mode as a function of Froude number.

The amplitude of the galloping mode is plotted in figure 11. Despite a strong scatter, the amplitude peak reaches values between one and two cylinder diameter. This is between two and four times the maximal value obtained for VIV modes (see figure 6 and compare figure 10 and figure 11).

The characteristics of this mode (low frequency and high amplitude oscillations) remind the classical galloping mode, which occurs in a homogeneous fluid when the axisymmetry of the object is broken. As shown for example by Bokaian \& Geoola (1984) in the case of a square cylinder, the galloping mode results from an instability: due to symmetry breaking, a tilt of the cylinder induces a lift in the same direction as the motion, and therefore increases the displacement. This instability leads to very large amplitude oscillations because they are only limited by the structure itself. Owing to its axisymmetry, no galloping instability appears for a circular cylinder in a homogeneous fluid. However, in the presence of stratification, as the axisymmetry is automatically broken, a destabilizing lift could a priori be generated even for a circular cylinder. Numerical simulations are presented in the next section to test this possibility.

\subsection{Numerical simulations}

Numerical simulations are performed using the commercial software COMSOL ${ }^{\circledR}$. The following configuration is simulated: in a fluid with a linear stratification $\bar{\rho}$, a cylinder is moved at a constant speed $\boldsymbol{U}$, in a direction tilted by an angle $\alpha$ with respect to the horizontal plane. The total density is written as $\rho=\rho_{0}+\bar{\rho}(z)+\rho^{\prime}(x, y, z, t)$ where $\rho_{0}+\bar{\rho}(z)$ is the background fluid density and $\rho^{\prime}$ the density induced by the cylinder displacement. Distances are non dimensionalized by $D$, velocities by $U$, pressure by $\rho_{0} U^{2}$ and density by $\rho_{0} D N^{2} / g$. In the cylinder frame of reference, the Navier-Stokes equations under the Boussinesq approximation and the density transport equation can then be written as

$$
\begin{aligned}
& \frac{\mathrm{D} \boldsymbol{u}}{\mathrm{D} t}=-\nabla p+\frac{1}{R e} \Delta \boldsymbol{u}-\frac{\rho^{\prime}}{F r^{2}} \boldsymbol{e}_{\boldsymbol{z}}, \\
& \frac{\mathrm{D} \rho^{\prime}}{\mathrm{D} t}-\left(u_{z}+\sin \alpha\right)=\frac{1}{R e S c} \Delta \rho^{\prime} .
\end{aligned}
$$

In equation $(5.1 \mathrm{~b})$, the $u_{z}$ term comes from the density transport due to the linear gradient, and the $\sin \alpha$ term is associated with the change of frame. 


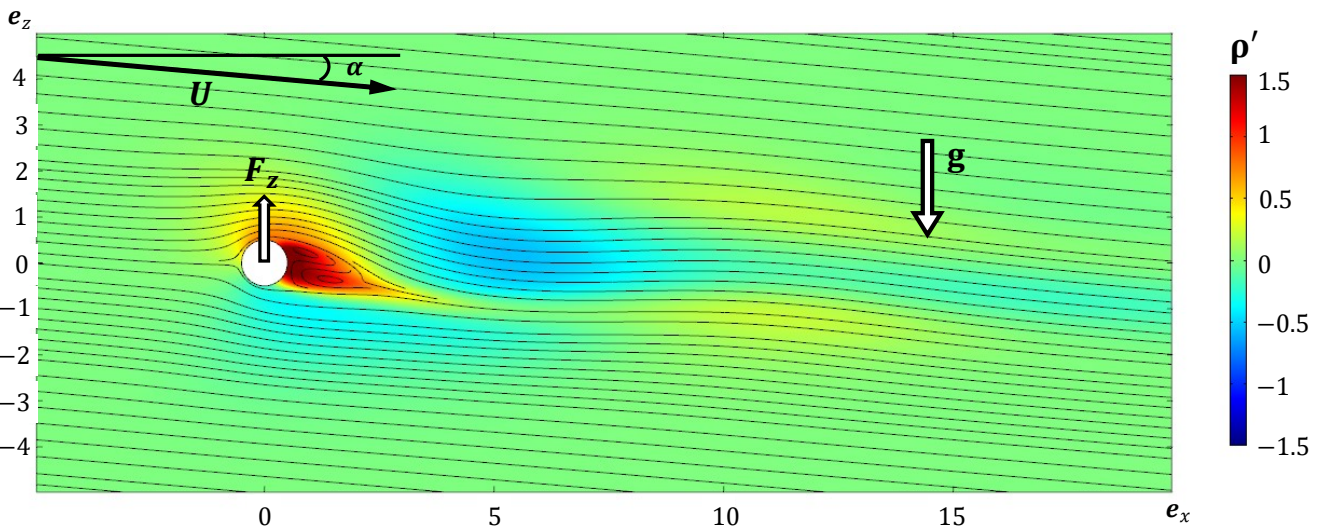

(a)

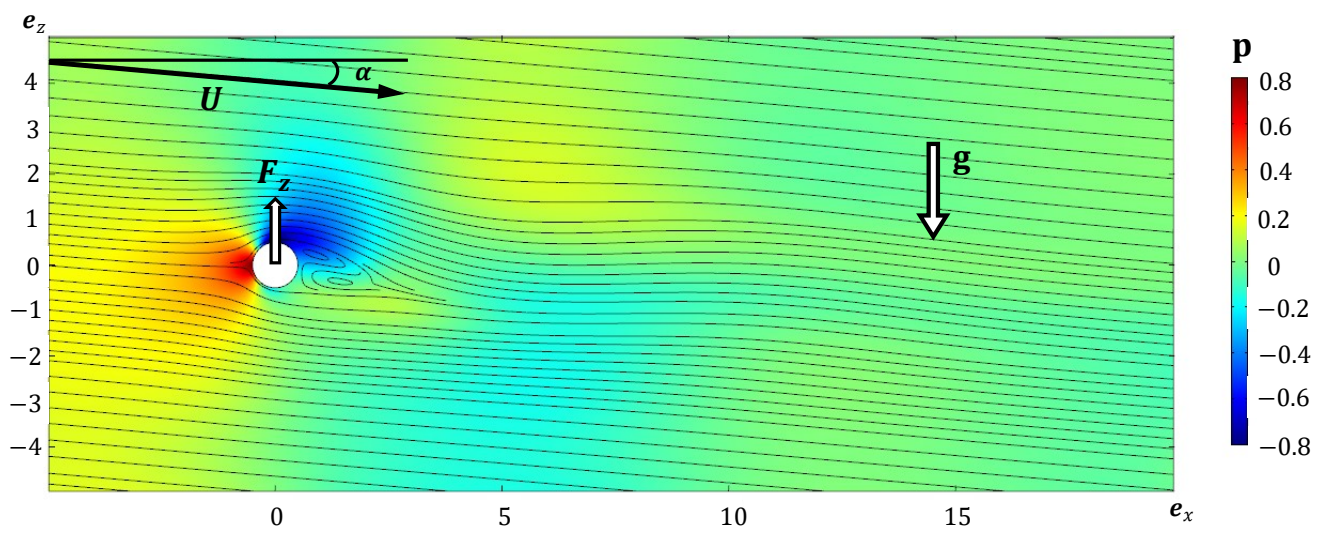

FiguRE 12. Properties of the stationary perturbation induced by an inclined displacement of the cylinder. Displacement angle with respect to the horizontal plane: $\alpha=5^{\circ} . F r=1.5, R e=300$, $S c=1$. (a): density field (color map) and streamlines. (b): pressure field (color map) and streamlines.

The problem is solved in a $(x, z)$ domain defined by $-5 \leqslant x \leqslant 20$ and $-5 \leqslant z \leqslant 5$ with the cylinder centred at the origin. The tilted velocity is imposed at the top $(z=5)$, the bottom $(z=-5)$ and the entrance $(x=-5)$ of the domain. Outlet boundary conditions are imposed at $x=20$. Smooth sponge layers of thickness 1 have been added at the top and bottom in order to damp internal waves: the viscosity is multiplied by a factor 1000 in these layers. A small Reynolds number $(R e=300)$ and a Schmidt number $S c=1$ are considered such that there is no vortex shedding. We are interested in the stationary solution to equations $(5.1 \mathrm{a}, \mathrm{b})$.

The density and pressure fields of this stationary solution are plotted in figure 12 . The resulting vertical force (sum of drag and lift components on $\boldsymbol{e}_{\boldsymbol{z}}$ ), shown in figure 13, is calculated by integrating the viscous force and pressure force on the cylinder.

Figure 12(a) shows that the density gradient is asymmetrically perturbed by the cylinder. A strong positive lobe of density is created above and behind the cylinder. This density correction is probably related to the combined action of the lee waves and of the recirculation zone. This positive density lobe induces an asymmetric pressure behind the cylinder since its $z$ derivative is equal to the density in the absence of motion. Indeed, although the usual positive pressure lobe causing drag is observed in front of the cylinder, figure 12(b) also demonstrates the presence of a negative pressure on the upper 


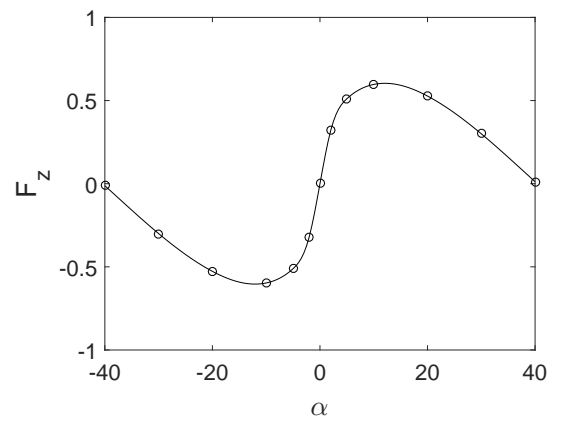

Figure 13. Evolution of the vertical force component $F_{z}$ as a function of the imposed tilt angle $\alpha . F r=1.5$ and $R e=300$.

right of the cylinder. This induces an upward lift. Since the cylinder is already going up, this lift reinforces the initial motion, thus an instability develops. Figure 13 shows that this destabilisation process occurs for angles in the range $\left[-40^{\circ}, 40^{\circ}\right]$. For larger angles, the drag force is stronger than the lift and the destabilizing mechanism described above is no longer present.

To conclude this section, it was shown by analysing the properties of the perturbation induced by the cylinder motion, that the lift can become destabilizing in the presence of stratification when the displacement is no longer horizontal. The mechanism is therefore very similar to the galloping instability.

The next section focuses on the influence of the non-dimensional parameters defined in section 2.3 on the development of the galloping and VIV modes.

\section{Competition between the two regimes}

As already mentioned, there are three main non-dimensional parameters characterizing the system: the length ratio $l$ which specifies the geometry, the Froude number $F r$ which compares buoyancy and inertial forces, and the Reynolds number $R e$ which characterises viscous effects. In this section, the influence of these different parameters on the observed mode is evaluated.

All data are plotted as a function of $F r$ and $l$ in figure 14(a). Each solid circle corresponds to an experiment where a distinct mode has been observed. The color of the circle is related to the frequency of the mode: red circles correspond to low-frequency galloping modes, while blue circles to high-frequency VIV modes. This stability map clearly shows that the galloping mode domain is limited to the square domain of high- $l$ and low-Fr: $l$ must be larger than 7 and $F r$ below 3 for the galloping mode to appear. However, these data cover a large range of Reynolds numbers.

To evaluate the influence of the Reynolds number, the data are plotted as a function of $F r$ and $R e$ in figure 14(b) for a reduced range of $l(l \in[8,12.5])$. The Reynolds number is found to have a weak impact on the transition between galloping and VIV mode compared to the two other non-dimensional numbers. The transition Froude number between the two modes seems to decrease as the Reynolds number increases. However it is difficult to quantify precisely the $R e$ dependence due the lack of data. Indeed, at transition, amplitudes are very weak and frequencies difficult to measure, so many experiments were not conclusive, causing a gap in the available data (see figure 14(b)).

Nevertheless, from figure 14, it can be inferred that the two main control parameters of the system are the Froude number and the geometric parameter $l$. This is in agreement 


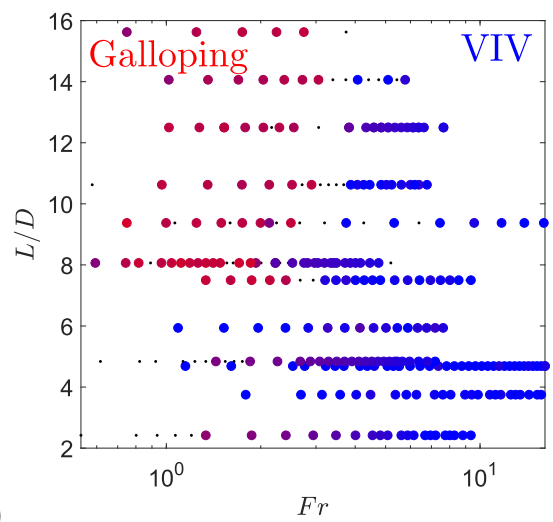

(a)
$\mathrm{Fr}$

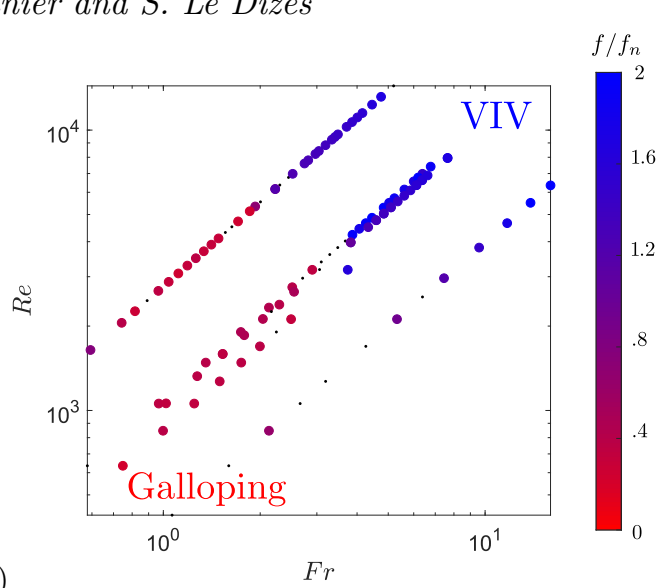

(b)

Figure 14. Experimental identification of galloping (in red) and VIV (in blue) modes based on the frequency measurement. Dots stand for performed experiments from which no frequency nor amplitude could be determined. (a) Map in $(F r, l)$ plane of all data. (b) Map in $(F r, R e)$ plane of a reduced set of data $(l \in[8,12.5])$.

with the model that we have developed. We have indeed shown that stratification is essential for the appearance of the galloping mode, while the parameter $l$ affects the drag force that is responsible for the development of the VIV mode. Viscosity is not expected to play an important role, especially in the regimes of large $R e / F r$ that we have considered.

\section{Conclusion}

The classical experiment of a spring-mounted cylinder has been adapted to the case of a stratified fluid: the flowing water channel has been replaced by a towing tank of stratified fluid, and the cylinder has not been mounted on springs but on flat bladeshaped arms free to rotate around their end. With this set-up, drag and buoyancy forces induce a vertical restoring force that mimics the action of a spring.

This pioneered experimental study of Vortex Induced Vibrations in a stratified fluid has brought out the existence of two different oscillating modes for a circular cylinder.

The first one is a VIV mode that results from the resonant coupling of vortex shedding with the natural oscillations induced by drag and buoyancy. It has a moderate amplitude (less than $0.6 D$ ) and a frequency proportional to the frequency $f_{n}$. The lock-in frequency ratio $f / f_{n}$ has been shown to be remarkably well-predicted by the classical VIV theory for low-mass-ratio (Williamson \& Govardhan 2004). The natural frequency is shown to be not constant. It varies from $f_{n} \sim N /(2 \sqrt{2} \pi)$ for small Froude numbers to $f_{n} \sim U / 2 \pi \sqrt{C_{D} / \pi L D}$ for large Froude numbers. In a spring-mounted experiment, this natural frequency would be expected to switch from $f_{n} \sim N /(2 \sqrt{2} \pi)$ to $\sqrt{k /\left(m^{*}+C_{A}\right)}$ as the spring constant $k$ increases.

The second mode is a galloping mode, characterized by a large amplitude (up to $2 D$ ) and a low frequency close to $0.25 N / 2 \pi$. It has been linked to the breaking of the axisymmetry due to the stratification. In particular, it has been associated with the presence of a destabilizing lift force, as for the galloping modes of non-cylindrical objects. This mode has been observed for low Froude numbers $(F r \leqslant 3)$, and long arms $(l \geqslant 7)$.

Galloping and VIV modes were also observed in classical VIV systems in a homogeneous fluid when the object is no longer axisymmetric, but the scenario of appearance 
is different. In general, as $U^{*}$ increases, VIV mode appears first, then it is replaced by the galloping mode, that never disappears (Nemes et al. 2012; Mannini et al. 2014). Here the scenario is opposite. We have first observed the galloping mode for small $U^{*}$ and then the VIV mode. This can be understood because small values of $U^{*}$ also correspond to small Froude numbers, which are necessary for the existence of the galloping mode. As $U^{*}$ increases, $\mathrm{Fr}$ also increases, so the buoyancy restoring force responsible for the instability of the galloping mode reduces, and is finally not sufficient to sustain the mode when $\mathrm{Fr}$ is too large.

The effect of the Reynolds number on the transition from galloping to VIV mode has been analysed for Re between 500 and 15000, but no significant dependence has been noticed.

Oceans, which are stratified, are full of cables (transatlantic communications, oil platforms...) subjected to complex streams, and then, potentially, FIV phenomenon. As this study shows a strong influence of stratification on fluid-structure interactions that has never been addressed before, it would be of interest to consider these specific applications. It suggests numerous possible studies. For instance, it would be useful to analyze the effects of an inclination angle, of the rigidity of the body and of a nonlinear stratification. Another setup with springs could also be useful for a comparison with classical homogeneous VIV results. Finally, using numerical simulations to explore regimes difficult to reach experimentally, such as low Froude numbers/large Reynolds numbers regimes could be another interesting research path.

The authors would like to thank Thomas Leweke for fruitful discussions.

\section{REFERENCES}

Assi, G. R. S., Bearman, P. W. \& Meneghini, J. R. 2010 On the wake-induced vibration of tandem circular cylinders: the vortex interaction excitation mechanism. J. Fluid Mech. 661, 365-401.

Bearman, P. W. 1984 Vortex shedding from oscillating bluff bodies. Annu. Rev. Fluid Mech. 16 (1), 195-222.

Bernitsas, M. M., Raghavan, K., Ben-Simon, Y. \& Garcia, E. M. H. 2008 VivaCE (Vortex Induced Vibration Aquatic Clean Energy): A new concept in generation of clean and renewable energy from fluid flow. J. Offshore Mech. Arctic Eng. 130 (4).

Blevins, R. D. 1977 Flow-induced vibration. New York, Van Nostrand Reinhold Co., $197 \%$. $377 \mathrm{p}$.

Bokaian, A. R. \& Geoola, F. 1984 Hydroelastic instabilities of square cylinders. J. Sound Vib. 92 (1), 117-141.

Bosco, M. 2015 Etude du sillage stratifié d'un cylindre. PhD thesis, Aix-Marseille.

Boyer, D. L., Davies, P. A., Fernando, H. J. S. \& Zhang, X. 1989 Linearly stratified flow past a horizontal circular cylinder. Phil. Trans. R. Soc. London. A 328 (1601), 501-528.

Chang, C.-C. J., Kumar, R. A. \& Bernitsas, M. M. 2011 Viv and galloping of single circular cylinder with surface roughness at $3.0 \times 10^{4} \leqslant r e \leqslant 1.2 \times 10^{5}$. Ocean Engineering 38 (16), $1713-1732$.

Chashechinin, Y. D. \& Voyekov, I. V. 1993 Vortex systems past a cylinder in a continuously stratified fluid. Izvestiya Atmos. Ocean. Phys. 29, 787-787.

Coutanceau, M. \& Bound, R. 1977 Experimental determination of the main features of the viscous flow in the wake of a circular cylinder in uniform translation. Part 1. Steady flow. J. Fluid Mech. 79 (2), 231-256.

Den Hartog, J. P. 1954 Recent technical manifestations of Von Karman's vortex wake. Proc. Nat. Acad. Sci. 40 (3), 155.

FENG, C. C. 1968 The measurement of vortex induced effects in flow past stationary and oscillating circular and d-section cylinders. PhD thesis, University of British Columbia. 
Govardhan, R. \& Williamson, C. H. K. 2000 Modes of vortex formation and frequency response of a freely vibrating cylinder. J. Fluid Mech. 420, 85-130.

GRIfFIn, O. M. 1985 Vortex-induced vibrations of marine cables and structures. Tech. Rep.. Naval Research Lab., Washington D. C.

Grouthier, C., Michelin, S., Bourguet, R., Modarres-Sadeghi, Y. \& De Langre, E. 2014 On the efficiency of energy harvesting using vortex-induced vibrations of cables. $J$. Fluids Struct. 49, 427-440.

Khalak, A. \& Williamson, C. H. K. 1999 Motions, forces and mode transitions in vortexinduced vibrations at low mass-damping. J. Fluids Struct. 13 (7-8), 813-851.

LiEnHARD, J. H. 1966 Synopsis of lift, drag, and vortex frequency data for rigid circular cylinders, , vol. 300. Technical Extension Service, Washington State University Pullman, WA.

Lin, J.-T. \& PaO, Y.-H. 1979 Wakes in stratified fluids. Annu. Rev. Fluid Mech. 11 (1), $317-338$.

Mannini, C., Marra, A. M. \& Bartoli, G. 2014 VIV-galloping instability of rectangular cylinders: Review and new experiments. J. Wind Eng. Ind. Aero. 132, 109-124.

Mannini, C., Marra, A. M., Massai, T. \& Bartoli, G. 2016 Interference of vortex-induced vibration and transverse galloping for a rectangular cylinder. J. Fluids Struct. 66, 403-423.

Meunier, P. 2012 Stratified wake of a tilted cylinder. Part 1. Suppression of a von Kármán vortex street. J. Fluid Mech. 699, 174-197.

Nakamura, Y., Hirata, K. \& Kashima, K. 1994 Galloping of a circular cylinder in the presence of a splitter plate. J. Fluids Struct. 8 (4), 355-365.

Nemes, A., Zhao, J., Lo Jacono, D. \& Sheridan, J. 2012 The interaction between flowinduced vibration mechanisms of a square cylinder with varying angles of attack. J. Fluid Mech. 710, 102-130.

Parkinson, G. V. \& WaWzonek, M. A. 1981 Some considerations of combined effects of galloping and vortex resonance. J. Wind Eng. Ind. Aerodyn. 8 (1-2), 135-143.

Roshko, A. 1961 Experiments on the flow past a circular cylinder at very high reynolds number. J. Fluid Mech. 10 (3), 345-356.

SARPKAYA, T. 2004 A critical review of the intrinsic nature of vortex-induced vibrations. $J$. Fluids Struct. 19 (4), 389-447.

Song, R., Shan, X., Lv, F. \& XIE, T. 2015 A study of vortex-induced energy harvesting from water using PZT piezoelectric cantilever with cylindrical extension. Ceramics International 41, S768-S773.

Strouhal, V. 1878 Über eine besondere art der tonerregung. Ann. Phys. 241 (10), 216-251.

Tritton, D. J. 2012 Physical fluid dynamics. Springer Science \& Business Media.

VON KARMan, T. 1911 Über den mechanismus des widerstandes, den ein bewegter körper in einer flüssigkeit erfährt. Nachr. Ges. Wiss. Göttingen, Math.-Phys. Kl. 1911, 509-517.

Williamson, C. H. K. 1996 a Three-dimensional wake transition. J. Fluid Mech. 328, 345-407.

Williamson, C. H. K. 1996b Vortex dynamics in the cylinder wake. Annu. Rev. Fluid Mech. 28 (1), 477-539.

Williamson, C. H. K. \& Govardhan, R. 2004 Vortex-induced vibrations. Annu. Rev. Fluid Mech. 36, 413-455. 